\title{
Status and Perspectives of the CBM experiment at FAIR
}

\author{
Norbert Herrmann ${ }^{1,2, *}$ for the CBM collaboration \\ ${ }^{1}$ Physics Institute, Heidelberg University, Germany \\ ${ }^{2}$ GSI Helmholtzzentrum für Schwerionenforschung GmbH, Darmstadt, Germany
}

\begin{abstract}
The CBM experiment, a fixed target experiment, designed to explore the QCD matter phase diagram in the baryon rich domain, is currently being constructed at the FAIR facility at GSI. The status of the construction towards the startup configuration of CBM and the initial physics goals are described. An outlook to the future physics perspectives is given.
\end{abstract}

\section{Overview}

The Compressed Baryonic Matter (CBM) experiment aims to study the QCD phase diagram in the baryon rich domain accessible by the SIS100 synchrotron of FAIR. The SIS100 machine with its rigidity of $100 \mathrm{Tm}$ will provide beams of protons and heavy nuclei up to $\mathrm{U}$. This enables measurements of heavy reaction systems like Au+Au up to a center-of-mass energy of $\sqrt{s_{N N}}=4.9 \mathrm{GeV}$. The energy range accessible by CBM will allow the detailed study of a possible first order phase transition as well as the location of the critical end point (CEP) according to recently published predictions $[2,3]$. Of particular interest to CBM is the high beam current and superb beam quality of SIS100 allowing the design of an apparatus that is capable of measuring rare probes with high statistics.

\footnotetext{
*e-mail: N.Herrmann@gsi.de
}

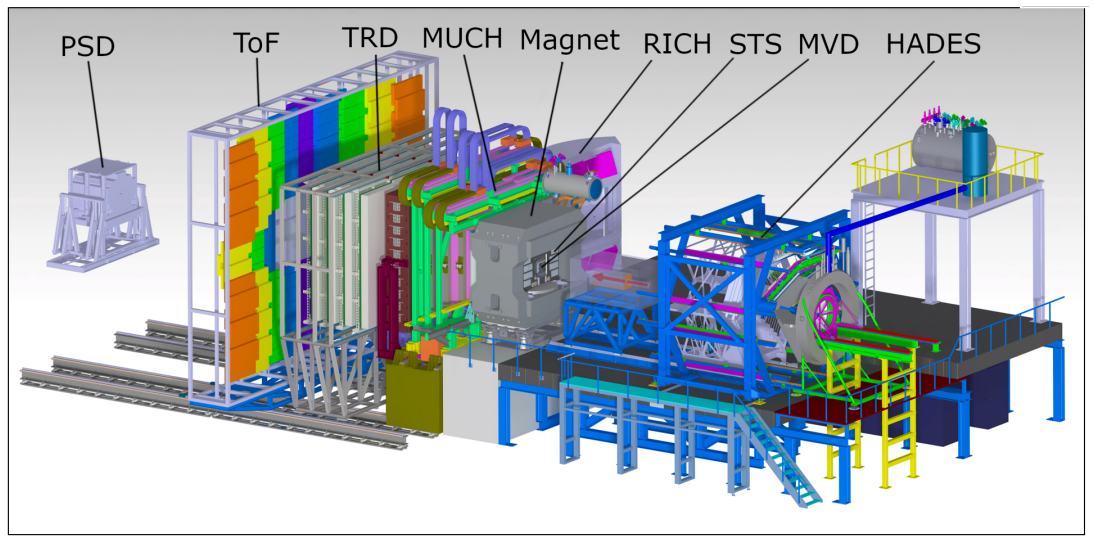

Figure 1. Setup of the CBM and HADES experiments sharing a common SIS100 beamline with different interaction regions. 


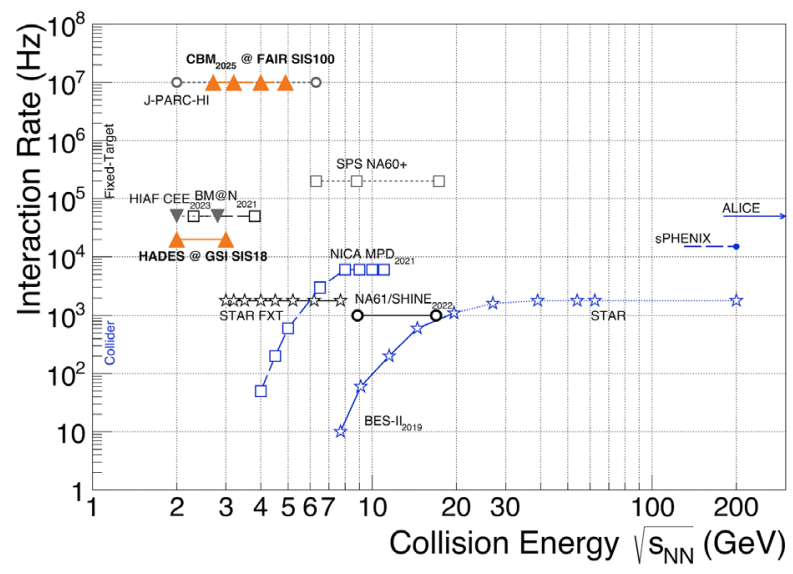

Figure 2. Comparison of the rate capability of experiments, Fig. updated from $[4,5]$

The layout of CBM with its different subsystems is shown in Fig.1 together with the HADES experiment [1] which will be installed along the same beamline but with a different target location. CBM is a general purpose forward spectrometer with a polar angle acceptance of $2.5^{\circ}<\theta<25^{\circ}$ and full azimuthal coverage. CBM can be operated in different configurations that are referred to as Hadron-Electron (HE) -, Muon (M) -, and Hadron-only (H) - setup. Common to all setups is the Silicon-Tracking-System (STS) that comprises 8 layers of double-sided micro strip Si-counters with a strip pitch of $58 \mu \mathrm{m}$ operated in a dipole magnetic field with a bending power of $1 \mathrm{Tm}$. This common STS tracker can be complemented by different subsystems enabling particle identification or optimizing topological signatures. These subsystems are shown in Fig. 1: Micro Vertex Detector (MVD), Ring Imaging Cerenkov Counter (RICH), MUon CHambers (MUCH), Transistion Radiation Detector (TRD), Time-Of-Flight system (TOF) and Projectile Spectator Detector (PSD). For combined electron and hadron identification the HE - setup makes use of MVD, STS, RICH, TRD, TOF and PSD, while the measurement of di-muons is done with the M - setup that implements an instrumented absorber and uses the subsystems STS, MUCH, TRD, TOF and PSD. Highest luminosity for identified hadron measurements is enabled by the $\mathrm{H}$ - setup employing STS, RICH, TRD and TOF. An important component not included in the figure is the data acquisition system of CBM. Since no hardware trigger is capable of reducing the event rate to a manageable level the full system is employing a trigger-less readout scheme. All detector signals are time stamped and forwarded by a high speed network to a high performance computing center where the event reconstruction and selection is done in software.

CBM and its subsystems are being designed to cope with interaction rates of heavy systems like $\mathrm{Au}+\mathrm{Au}$ up to a peak rate of $10 \mathrm{MHz}$ with full event reconstruction. This feature is so far not reached by any other experiment, a comparison to other facilities is shown in Fig. 2.

\section{Site preparation and experiment status}

With the restart of FAIR in 2017, the progress in construction and civil engineering is impressive as can be seen from Fig.3 [6]. After a rebaselining process in 2021 first beams from SIS100 are now expected for 2026. The timeline for CBM has been adjusted accordingly. The CBM building will be finished in 2022 and equipped with heavy infrastructure like the magnet foundation and the rail system. In 2023 the magnet will be delivered and all the services 


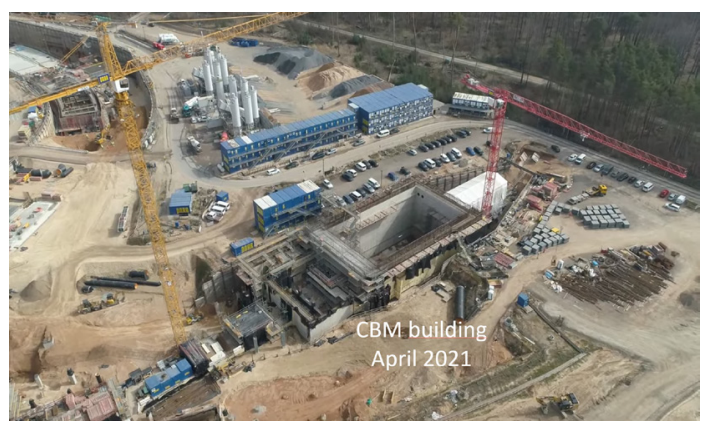

Figure 3. Status of civil construction of the CBM cave as of April 2021. The walls and the beam dump are finished, the roof is planned to be done in Q3, 2021. Aerial photography was extracted from a video of a drone fly over the FAIR construction site in April 2021 [6].

will be installed. Detector installation is planned for 2024 ending with the commissioning phase that is anticipated for 2025 .

Almost all the CBM subsystems have concluded their R\&D phases and either have already built the entire detector (PSD) or are preparing for series production (STS, TRD, RICH, MUCH and TOF). The MVD comprising 288 sensors with $148 \mathrm{M}$ pixels in MAPS technology based on the Tower Jazz $180 \mathrm{~nm}$ process is also at its final evaluation steps and recently submitted its Technical Design Report (TDR). Many of the subsystems have had major milestones successfully completed recently. For the magnet, the acceptance of Production Readiness Review (PRR) for the yoke and the power supply marks the start of production. The STS has already all its sensors produced and tested for acceptance. The current focus is on integration of prototype modules and ladders with the final readout ASIC (STS-XYter 2.2). In addition thermal stability issues are now addressed since the sensors will be operated below a temperature of $-5^{\circ} \mathrm{C}$. MUCH has procured all the necessary GEM foils and is on the track for the Engineering Design Review (EDR) of its mechanics. With a successful operation of their MAPMT sensors in the HADES experiment, RICH is now focussing on the camera design and its mechanics. The TRD subsystem passed its PRR for the outer chamber production and at the same time submitted a TDR for a modified design of the inner readout chambers which will enhance the tracking capability by a 2-dimensional position measurement and will increase the CBM acceptance for charged particles to vanishing transverse momenta at mid-rapidity. The TOF system employing MRPC detectors is pushing for the determination of the rate limitations of their counters that use different technologies for the floating electrodes (see section 5). In addition aging test have been performed at the ISRAM facility in Bucharest. The PSD subsystem is most advanced along its timeline as demonstrated by the delivery of their support structure to FAIR. All calorimeter modules are produced and used in the framework of FAIR phase-0 in the BM@N and NA61 experiments.

Further details about the detector technologies of the subsystems can be found in the TDRs that are publicly available [7]. In summary, prototype or pre-series modules are available for all subsystems. Some CBM detectors are already used in running physics experiments, PSD modules are operated in NA61 (CERN) and BM@N (JINR) and TOF modules are contributing to BES-II data taking in STAR (BNL). All of the subsystems are present in CBM's own demonstrator, the mini-CBM (mCBM) experiment (see section 5).

\section{Physics program}

The overall physics program of CBM is outlined in [4] and addresses all topics relevant for the characterization of dense baryonic matter. As mentioned above a highlight of CBM is the possibility to correlate bulk observables of the reactions with the abundance of rare probes. According to current full system simulations, bulk properties like centrality and 


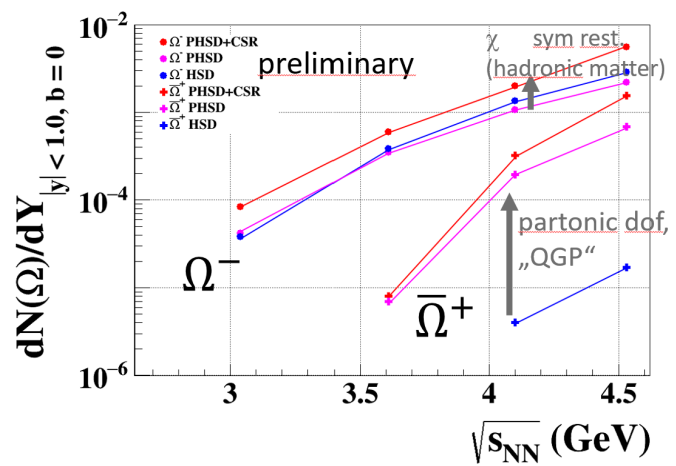

Figure 4. Prediction of $\Omega$ and $\bar{\Omega}$ production in the transport models HSD and PHSD $[8,9]$, for details see text.

collective flow can be well reconstructed in the forward hemisphere of the reaction. The rare probes whose phase space distribution appear to be accessible to CBM at SIS100 are multistrange baryons, di-electrons, di-muons, $\mathrm{J} / \Psi$ and single and double $\Lambda$ - hypernuclei. With this menu of observables, new insights are expected for the Equation-Of-State (EOS) of dense baryonic matter with its relevance for the stability of neutron stars, the phase structure of the QCD phase diagram, the existence of a CEP, chiral symmetry restoration and in-medium modifications of hadrons, strange matter and the interaction of heavy quarks with cold dense matter. In addition, the experiment is well suited to search for exotic QCD states like multistrange di-baryons, strange penta-quarks, multi- $\Lambda^{*}$ systems, double $\Lambda$ hypernuclei and the like. To which extent the production of these objects is enabled or favored by the dense baryonic environment is an interesting research topic that will be pursued in the future. All of these programs will need systematic measurements in terms of excitation functions and characterization of centrality and reaction plane orientation. To obtain significant results, a high rate experiment like CBM is indispensable.

\section{Day-1 measurements}

Since the ultimate rates, which the experiment is designed for, will not be available at the beginning of SIS100 operation the experiment will start in 2025 with its so-called day-1 configuration, the HE - and the M - setup. Despite an initial rate limitation of $10^{5}$ interactions per second due to the readout speed of the MVD, important measurements may be obtained from these setups. For example, the excitation function of the production probability of multiple-strange baryons and the one excitation of di-lepton production may be measured. Note that the CBM day-1 rate exceeds the ones of all currently running experiments (c.f. Fig.2).

As an example, Fig. 4 depicts the expectations of $\Omega$ and $\bar{\Omega}$ production yields for a possible outcome of the measurement in the framework of a transport model [8] where different assumptions on the underlying physics were implemented. It can be seen that a strong increase of the $\bar{\Omega}$ is obtained when partonic degrees of freedom (string contributions) are allowed. While the effect of partonic degrees of freedom on the $\Omega$ production is rather small, both species are affected by the hadronic phase that is modeled to include chiral symmetry restoration (CSR). The measurement of the strange baryon rates with sufficient statistics will allow the discrimination of the scenarios. Full system simulations were performed and a highly efficient particle reconstruction package ("KFParticle") was developed and applied. Reconstruction efficiencies for $\Omega(\bar{\Omega})$ in the $\mathrm{Au}+\mathrm{Au}$ reaction at an incident energy of $\sqrt{s_{N N}}=4.5 \mathrm{GeV}$ were determined to $5 \%(3 \%)$. Within a week of data taking about $10^{6}\left(10^{5}\right) \Omega(\bar{\Omega})$ parti- 

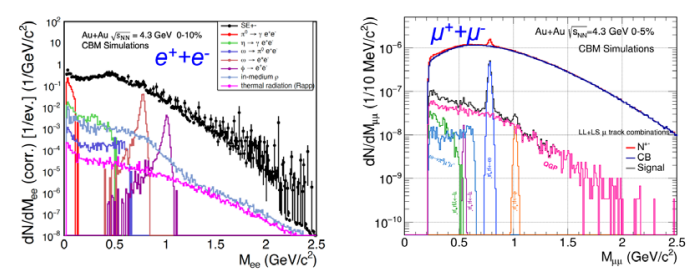

Figure 5. Simulated invariant mass spectra of $e^{+} e^{-}$(left) and $\mu^{+} \mu^{-}$(right) at an incident energy of $\sqrt{s_{N N}}=4.3 \mathrm{GeV}$ obtained with the corresponding day-1 setups, HE and M, respectively

cles will be reconstructed at this energy if the scenario "PHSD" is assumed. This allows a significant distinction from the "HSD" scenario which predicts only $2 \cdot 10^{3}$ reconstructed $\bar{\Omega}$ - baryons. A high statistics measurement will be able to differentiate among the various assumptions that are admittedly different in different models.

Figure 5 presents the current status of simulations for the invariant mass of lepton pairs at an incident energy of $\sqrt{s_{N N}}=4.3 \mathrm{GeV}$. Both setups show clear peaks of the $\omega$ - meson and a similar signal-to-background ratio. An advantage of the SIS100 energy range for the study of di-leptons with respect to higher beam energies is the negligible contribution from Drell-Yan process and from open charm decays. With the current material budget and detector performance, the most demanding goal to determine the slope of the invariant mass spectrum in the intermediate mass region (IMR), i.e. invariant mass range from $1.1 \mathrm{GeV}$ to $3 \mathrm{GeV}$, seems to be possible. This region is specifically interesting since the slope of the spectra provides access to the initial fireball temperature in the dense phase of the reaction and the yield is carrying information about chiral symmetry [10]. However, such a measurement needs large statistics to cope with the small signal-over-background ratio. To reach a statistical accuracy of $10 \%$ for the slope parameter with the HE - setup about $10^{11}$ events have to be recorded for which a total time of about 20 days will be needed. The comparison of the spectra from dielectron and di-muons will allow the systematic uncertainty to be assessed. CBM is therefore well prepared to do systematic measurements with these penetrating probes to diagnose the in-medium properties of hadrons and eventually quantify chiral symmetry restoration or even find a signal for a phase transition [4].

\section{5 mCBM experiment}

In order to prepare for its challenging program, $\mathrm{CBM}$ has constructed and installed a setup called mini CBM (mCBM) that allows tests of pre-series and prototype detectors under realistic conditions using high intensity SIS18 beams. In addition, mCBM also serves a demonstrator and development site of the free streaming data acquisition and online data analysis chain including necessary tools for controls and monitoring. A picture of the setup is shown in Fig. 6. For a trigger-less data acquisition system as under development for CBM, it is mandatory that all front-end devices that provide time stamps to the data messages are and stay synchronized even in the presence of high load from misbehaving detector components throughout the data taking. This important step could be verified during test beam campaigns in 2020 and 2021, the successful time synchronization is visible in Fig. 6 (right, bottom) where the time difference to a common reference is shown. Building on the synchronized data stream spatial correlations of the detector subsystems MUCH, RICH and TRD with reference tracks provided by the TOF subsystems (as depicted in the upper right panel of Fig. 6) have been observed and are currently being analyzed to extract detection efficiencies. Interaction rate of up to $1 \mathrm{MHz}$ and charged particle fluxes of up to $20 \mathrm{kHz} / \mathrm{cm}^{2}$ at a distance of $3 \mathrm{~m}$ from the target have been reached and could be handled by the detectors. As a next step 

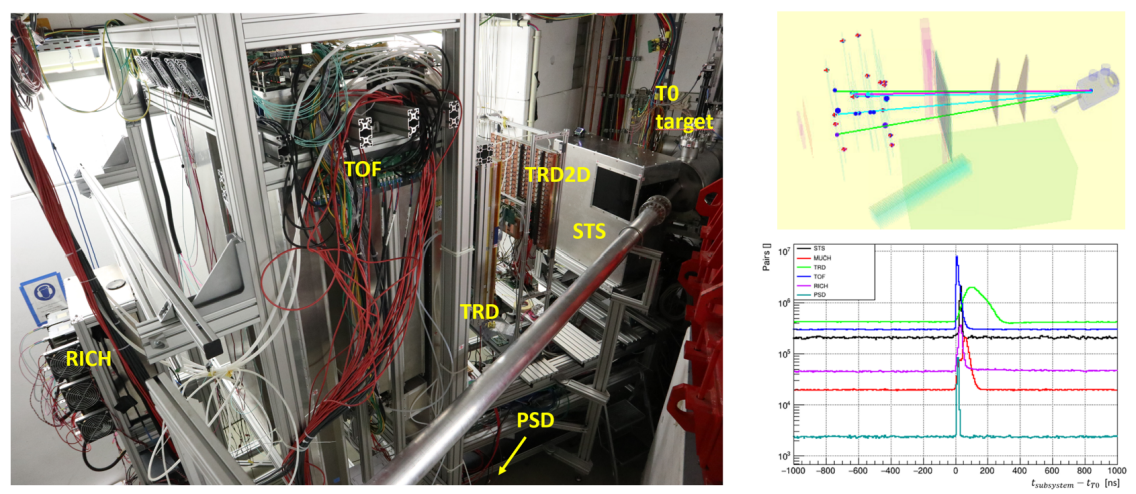

Figure 6. Photo of the mCBM setup with subsystem labels (left), event display with reconstructed TOF tracks (right, top) and time correlation of all subsystems with respect to the beam counter (right, bottom)

for full mCBM the reconstruction of $\Lambda$ - baryons is planned with the perspective to measure the excitation function in the sub-threshold region.

\section{Conclusion and Outlook}

$\mathrm{CBM}$ as a high rate experiment has a unique physics potential in mapping out the properties of rare probes originating from the compressed phase of baryonic matter produced in heavyion reactions. The preparation of the experiment is advancing well towards the day- 1 setups that will be ready to take first collision data in 2025 .

\section{Acknowledgments}

This work is supported by funding agencies of Germany (BMBF 05P15VHFC1, 05P19PMFC1, 05P19PXFCA, 05P19RFFC1, 05P19RGFCA, DFG 405580046, GSI, HIC for FAIR, HGS-HIRe, HFH FAIR, FIAS), European Union (Horizon 2020 no.871072 CREMLINplus), Russia (MSHE of RF 0723-2020-0041, RFBR 18-02-40086, RSF 17-7220234), India (VECC EHEP and A-Group, University of North Bengal), Poland (WUT IDUB-POB-FWEiTE-1), China (NNSF 11927901, 11420101004, 11735009, U1832118, MST 2018YFE0205200, 2016YFA0400100), Czech Republic (CZ.02.1.01/0.0/0.0/16 013/0001677, LM2018112), and Romania (MRID NUCLEU PN19060103, FAIR03/16.11.2020). The mCBM experiment in carried out within the framework of the FAIR phase-0 program.

\section{References}

[1] G. Agakichiev et al., Eur. Phys. J. A 41 (2009) 243

[2] W. Fu, J. Pawlowski, F. Rennecke, Phys.Rev. D 101 (2020) 5

[3] F. Karsch, arXiv:1905.03936

[4] T. Ablyazimov et al. (CBM Collaboration), Eur. Phys. J. A 53 (2017) 60

[5] T. Galatyuk, Nucl.Phys. A 982 (2019) 163

[6] https://www.youtube.com/watch?v=btwRHqcVW4A 
[7] https://www.cbm.gsi.de/documents

[8] W. Cassing et al., Phys.Rev. C 93 (2016) 014902

[9] I. Vassiliev, priv. comm.

[10] R. Rapp, H. v.Hees, Phys.Lett. B 753 (2016) 586 